\title{
Computer Simulation of Sandwich Sheet Metal Forming
}

\author{
Igor A. Brigadnov \\ Department of Computer Science \\ National Mineral Resources University "Gorniy" \\ St. Petersburg, Russian Federation \\ E-mail: brigadnov@mail.ru
}

\begin{abstract}
Large elastic-plastic deformations of sandwich composite sheets are computer simulated. Within a framework of the shell theory, the elastic-plastic constitutive relation for transversely anisotropic sandwich sheet taking into account of the Bauschinger effect is discussed. Results of the computer simulation of a hemispherical punch stretching operation are demonstrated.
\end{abstract}

Keywords: Sandwich sheet, large elastic-plastic deformations, hemispherical punch stretching, computer simulation.

\section{Introduction}

Stamping of sheet metal parts by means of hard punches and dies is a standard manufacturing process. Despite its broad application in car-industry, however, sheet metal stamping technology is still an art rather than a science. Thus the design of stamping process and the selection of sheet materials for a practical product are largely done on a trial-and-error basis.

In computer simulation of sheet metal forming, several finite element methods such as membrane, solid and shell model are proposed. The membrane model is not applicable where the bending of sheets becomes significant, for example, in drawing operations. In general, the processes where the bending effect is significant are simulated by the solid model or the shell model. For the solid model, more elements are needed to describe these shell-type structures to prevent numerical difficulty so that a large system of equations must be solved. The shell model is more effective than the solid one although integration in the thickness direction is still needed. 
Here we present the paper in the following order. First, we present the results of the standard uniaxial stretch experiments for aluminum-polypropylene-aluminum sandwich sheets named by the Hoogovens Hylite BV as Hylites (see Fig. 1). After that we describe the elastic-plastic $2 \mathrm{D}$ constitutive relation for transversely anisotropic sandwich sheet and present a brief description of axisymmetric stamping experiments for Hylites. At the end, the punch force for Hylites is numerically investigated under the hemispherical punch stretching operation.

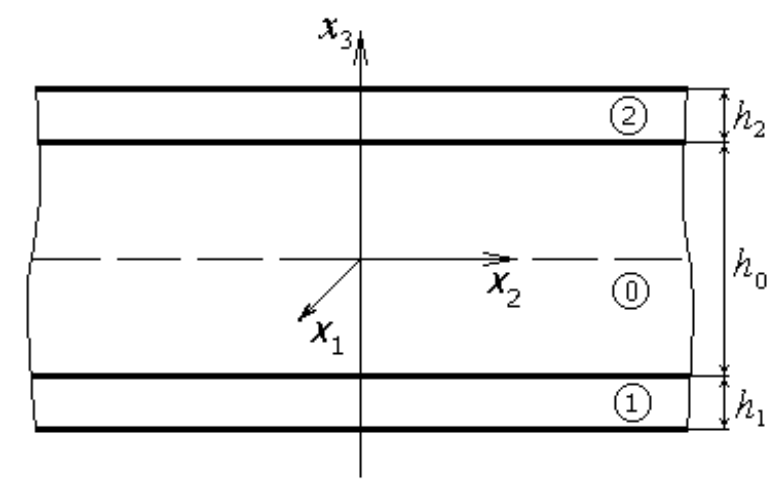

Figure 1: The three-layer sandwich sheet.

\section{Experimental Research of Hylites}

The following standard Hylite sandwich sheets were researched (Fig. 1): skin material (layers 1 and 2) is aluminum AA5182 of thickness 0.20-0.25 ( $\mathrm{mm}$ ), softannealed (deep drawable) or full hard; core material (filler 0) is polypropylene.

In experiments the basic mechanical parameters of sandwich sheets have been measured in the classical uniaxial stretch experiments with standard specimens. All experiments were realized on the test machine INSTRON-1253 by the standard methods at the Department of Special Tests of Central Test Laboratory, which is the part of the Center of Metallurgical Technologies and Research in "Izhorskiye Zavody" (ISC - Izhorsky Stock Company, Kolpino, St. Petersburg).

The main mechanical parameters for 5 types of Hylites are presented in Table 1, where $\sigma_{y} \approx \sigma_{0.2}$ is the initial yield stress, $\sigma_{S}$ is the tensile strength and $E$ is the Young modulus. The following accuracy of measurements was guaranteed: for stress $1 \%$, for displacement $0.01 \mathrm{~mm}$ and for linear sizes $0.1 \mathrm{~mm}$.

From the theory of multi-layer sheets it follows that the Young integral modulus and mass density can be calculated by relations

$$
E=\sum_{k=0}^{2} E_{k} h_{k} / \sum_{k=0}^{2} h_{k}, \quad \rho=\sum_{k=0}^{2} \rho_{k} h_{k} / \sum_{k=0}^{2} h_{k},
$$

where $E_{k}, \rho_{k}$ and $h_{k}$ is the Young modulus, the mass density and the thickness of $k$-layer in Fig. 1. For all Hylites we have $h_{1}=h_{2}$. It is well known that for 
aluminum the Young modulus is approximately 69-71 $G P a$ and for polypropylene it is approximately 1400-1600 $M P a$. Using the first relation (1), it is easily verified that for sandwich sheets $\mathrm{H} 1$ and $\mathrm{H} 2$, the Young modulus is approximately $24 \mathrm{GPa}$ and for $\mathrm{H} 4$ and $\mathrm{H} 5$ it is approximately $15 \mathrm{GPa}$. Just these theoretical values are presented in the Product Information of Hoogovens Hylite BV, where information about Hylite sheet $\mathrm{H} 4$ as well as information about integral yield stresses for all Hylites is fully absent.

Table 1: The main mechanical parameters for 5 types of Hylites

\begin{tabular}{|c|c|c|c|c|c|c|}
\hline $\begin{array}{c}\text { ID } \\
\text { name }\end{array}$ & $\begin{array}{c}\text { Thickness } \\
(\text { total/core })\end{array}$ & $\begin{array}{c}\text { Skin } \\
\text { (aluminum) } \\
\text { characteristics }\end{array}$ & $\begin{array}{c}\text { Mass } \\
\text { density } \\
\left(\mathrm{g} / \mathrm{cm}^{3}\right)\end{array}$ & $\begin{array}{c}\sigma_{y} \\
(\mathrm{MPa})\end{array}$ & $\begin{array}{c}\sigma_{S} \\
(\mathrm{MPa} a)\end{array}$ & $\begin{array}{c}E \\
(\mathrm{GPa} a)\end{array}$ \\
\hline $\mathrm{H} 1$ & $1.2 / 0.8$ & $\begin{array}{c}\text { soft skins EDT } \\
\text { surface }\end{array}$ & 1.52 & 53.0 & 101.0 & 25.56 \\
\hline $\mathrm{H} 2$ & $1.2 / 0.8$ & hard skin & 1.52 & 131.0 & 147.0 & 27.64 \\
\hline $\mathrm{H} 3$ & $1.4 / 0.92$ & $\begin{array}{c}\text { soft skin EDT } \\
\text { surface }\end{array}$ & 1.53 & 54.6 & 100.0 & 25.96 \\
\hline H4 & $2.0 / 1.6$ & hard skin & 1.28 & 86.0 & 98.0 & 18.53 \\
\hline H5 & $2.4 / 1.9$ & soft skin & 1.29 & 36.6 & 70.7 & 18.05 \\
\hline
\end{tabular}

It is well known that the mass density of aluminum is $\rho_{1}=\rho_{2} \approx 2.7\left(\mathrm{~g} / \mathrm{cm}^{3}\right)$ and the mass density of polypropylene is $\rho_{0} \approx 0.92 \div 0.93\left(\mathrm{~g} / \mathrm{cm}^{3}\right)$. In Table 1 the calculated mass densities for all Hylites are presented. We have more realistic experimental results for the Young integral modulus and other two main integral parameters for Hylites which were used in the hemispherical punch stretching experiments and the appropriate computer simulations.

From our experimental research it follows that Hylites have a small linear strain hardening with current Young's integral modulus $E_{t}<<E$. For all Hylites the parameter of strain hardening $K=E_{t} / E \approx(1.7 \div 1.8) \cdot 10^{-3}$, therefore, we recommend to use in practice the model of ideal (perfect) elastic-plasticity with $K=0$.

Mechanical properties of polypropylene essentially depend on temperature [1]. It is well known that the polypropylene is really incompressible. As a result, it's Poisson's ratio $v=0.5$ and the shear modulus $\mu=E / 3$. From the statistical physics we have the following relation for the polypropylene shear modulus $\mu=N k T$, where $N$ is the number of macro molecules in the unique volume, $k \approx 1.38 \cdot 10^{-23}(N \cdot m / K)$ is the Boltzmann constant and $T$ is the absolute temperature. The Young modulus of polypropylene is $E_{0} \approx 1500 \mathrm{MPa}$ for the room temperature $t=25^{\circ} \mathrm{C}$, i.e. for the absolute temperature $T_{0}=298 \mathrm{~K}$. As a 
result, we have the simplest relation for the polypropylene shear modulus $\mu=\mu_{0}\left(T / T_{0}\right) \approx 1.7 \cdot T$, where $\mu_{0} \approx 500 \mathrm{MPa}$ and $T \leq T_{*}=418 \mathrm{~K}\left(145^{\circ} \mathrm{C}\right)$.

\section{The Shell Yield Function for Transversely Anisotropic Sheets}

Consider Cartesian reference axes which are parallel to the three symmetry planes of anisotropic behavior. Then, the Hill yield function [2,3] can be written as

$$
F\left(\sigma_{22}-\sigma_{33}\right)^{2}+G\left(\sigma_{33}-\sigma_{11}\right)^{2}+H\left(\sigma_{11}-\sigma_{22}\right)^{2}+2 L \sigma_{23}^{2}+2 M \sigma_{31}^{2}+2 N \sigma_{12}^{2}-1=0,
$$

where the constants $F, G, H, L, M, N$ are related to the yield stresses by

$$
\begin{gathered}
2 L=\frac{1}{\sigma_{y 23}^{2}}, \quad 2 M=\frac{1}{\sigma_{y 31}^{2}}, \quad 2 N=\frac{1}{\sigma_{y 12}^{2}}, \quad 2 F=\frac{1}{\sigma_{y 2}^{2}}+\frac{1}{\sigma_{y 3}^{2}}-\frac{1}{\sigma_{y 1}^{2}}, \\
2 G=\frac{1}{\sigma_{y 3}^{2}}+\frac{1}{\sigma_{y 1}^{2}}-\frac{1}{\sigma_{y 2}^{2}}, \quad 2 H=\frac{1}{\sigma_{y 1}^{2}}+\frac{1}{\sigma_{y 2}^{2}}-\frac{1}{\sigma_{y 3}^{2}} .
\end{gathered}
$$

Here $\sigma_{y 1}, \sigma_{y 2}$ and $\sigma_{y 3}$ are the tensile yield stresses and $\sigma_{y 12}, \sigma_{y 23}$ and $\sigma_{y 31}$ are the shear yield stresses. For the isotropic von Mises plasticity all yield stresses equal to $\sigma_{y}$ and, therefore, we have constants

$$
F=G=H=\frac{1}{2 \sigma_{y}^{2}}, \quad L=M=N=\frac{3}{2 \sigma_{y}^{2}} .
$$

For computer simulation of sandwich sheet stamping process within a framework of the shell 2D model we use the transverse anisotropy, where properties do not vary in the $x_{1}-x_{2}$ plane (see Fig. 1). In this case it is assumed that $\sigma_{y 1}=\sigma_{y 2}=\sigma_{y}$ and the following relations hold:

$$
2 F=2 G=\frac{1}{\sigma_{y 3}^{2}}, \quad 2 H=\frac{2}{\sigma_{y}^{2}}-\frac{1}{\sigma_{y 3}^{2}}, \quad 2 N=\frac{4}{\sigma_{y}^{2}}-\frac{1}{\sigma_{y 3}^{2}} .
$$

Therefore, using the parameter $K=\sigma_{y} / \sigma_{y 3}$, the yield criteria can be written in the form $f(\sigma)=\sigma_{y}$, where

$$
\begin{aligned}
f^{2}(\sigma)= & \sigma_{11}^{2}+\sigma_{22}^{2}+K^{2} \sigma_{33}^{2}-K^{2} \sigma_{33}\left(\sigma_{11}+\sigma_{22}\right)-\left(2-K^{2}\right) \sigma_{11} \sigma_{22}+ \\
& +2\left(\sigma_{23}^{2}+\sigma_{31}^{2}\right)+\left(4-K^{2}\right) \sigma_{12}^{2}
\end{aligned}
$$


The rate of plastic strain is assumed to be normal to the yield surface [1-5]:

$$
D_{i j}^{p}=\lambda \frac{\partial f}{\partial \sigma_{i j}} .
$$

Here $D^{p}$ is the plastic part of the strain rate $D=\left(L+L^{T}\right) / 2$, where $L$ is the gradient of material derivative of displacement in the current (actual) configuration.

We define the anisotropy parameter $R$ as the ratio of in-plane plastic strain rate to the out-of-plane plastic strain rate $R=D_{22}^{p} / D_{33}^{p}$ such that $R=1.0$ for the isotropic case. It then follows that $R=2 K^{-2}-1$. Using the plane stress assumption and the definition of $R$, as a result, the Hill yield function can be written in the following simplest form:

$$
f^{2}(\sigma)=\sigma_{11}^{2}+\sigma_{22}^{2}-2\left(\frac{R}{R+1}\right) \sigma_{11} \sigma_{22}+2\left(\frac{2 R+1}{R+1}\right) \sigma_{12}^{2} .
$$

\section{Taking into Account of the Bauschinger Effect}

The back stress $s$, which prescribes the position of the center of the yield surface in the stress space, provides the asymmetry in the yield function between continued and reversed loading needed to incorporate such phenomena as the Bauschinger effect $[4,5]$.

For combined kinematic-isotropic hardening with the isotropic-hardening stress measure satisfying the von Mises type yield condition, the yield function takes the following form $[4,5]$ :

$$
(\tau-s):(\tau-s)=2 \sigma_{y}^{2}
$$

where $\tau=\sigma-\frac{1}{3} \operatorname{tr}(\sigma) I$ is the deviator of the Cauchy stress tensor $\sigma, I$ is the identity second-order tensor, symbol $\operatorname{tr}(\sigma)$ corresponds to the trace of the tensor $\sigma$ and $\sigma_{y}$ is the initial yield stress. The latter depends on the history of plastic deformation as expressed through the generalized plastic strain scalar $\lambda$ from (3) given by the growth law

$$
\lambda=\sqrt{D^{p} \cdot \cdot D^{p}}
$$

The growth of the anisotropic part of yield stress in kinematical hardening is given by the evolution equation [5] 


$$
\dot{s}^{*}=C(\lambda) D^{p}
$$

where for finite-deformation applications, the objective derivative $\dot{s}^{*}$ is commonly chosen to replace the material derivative \& used in infinitesimal displacement theory. This ensures that (5) is objective under superposed timedependent rigid body rotations.

From the physical point of view the Truesdell-Oldroyd rate $\dot{a}^{T r}=\dot{a}-L \cdot a-a \cdot L^{T}$ for the tensor $a$ is the most realistic [5]. Therefore, this rate can be recommended for using in all constitutive relations of materials within a framework of the finitedeformation theory taking into account the Bauschinger effect. As a result, for the ideal Bauschinger effect, i.e. for $C \equiv 2 \mu$ in (5), we have the following relation:

$$
\dot{s}^{T r}=2 \mu D-\dot{\tau}^{T r},
$$

because Hooke's law for objective rates $\dot{\tau}^{T r}=2 \mu\left(D-D^{p}\right)$ is true [2-5].

As a result, the generalized yield function for Hylites without isotropic hardening, i.e. for the perfect plasticity, and the ideal Bauschinger effect has the following simplest form [6]:

$$
f^{2}(\rho)=\rho_{11}^{2}+\rho_{22}^{2}-2\left(\frac{R}{R+1}\right) \rho_{11} \rho_{22}+2\left(\frac{2 R+1}{R+1}\right) \rho_{12}^{2},
$$

where $\rho=\tau-s$. Here $R$ is unknown parameter of anisotropy which can be defined by the correlation between physical experiment and computer simulation.

\section{Experimental Research of Hemispherical Punch Stretching}

A hemispherical punch stretching operation is schematically shown in Fig. 2. The punch, binder and die are rigid (medical still). A piece of sandwich sheet is pressed by a hemispherical punch through a circular arc-flanged die. The geometric parameters of the punch stretching operation are:

- $r_{p}=50.8(\mathrm{~mm})$ is the punch profile radius,

- $r_{d}=6.35(\mathrm{~mm})$ is the die profile radius,

- $r_{0}=53.23(\mathrm{~mm})$ is the punch opening radius,

- $h_{0}=1.2 \div 2.4(\mathrm{~mm})$ is the sheet thickness.

The punch moves vertically by the rigid kinematical press with a registration of its displacement $d$. The punch force $P$ is measured by the original piezometer setting between the press and punch. The following accuracy of measurements is guaranteed:

- for the punch force $2 \%$, 
- for the punch displacement $0.1 \mathrm{~mm}$.

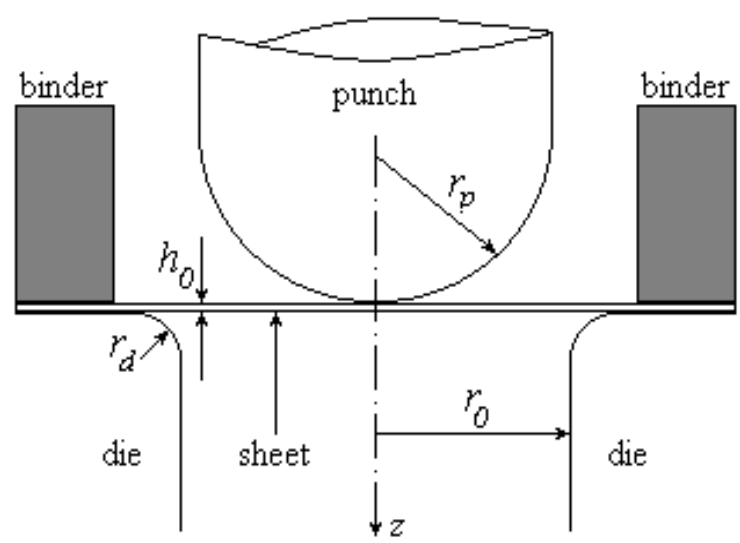

Figure 2: A hemispherical punch stretching operation.

In this type of sheet stretching operation, the central area of punch contacts the sheet first, and then the contact region expands. As the contact region expands, combined bending and stretching deformation gradually changes to stretchingdominated deformation. The stress resultant states under stretching-dominated conditions are close to the vertices that exist along the pure stretching direction in the stress resultant space.

In our experiments the punch force $P$ as the function of the displacement $d$ was received. For Hylites $\mathrm{H} 1$ and $\mathrm{H} 2$ the appropriate experimental curves 1 are presented in Fig. 3.a and 3.b respectively.

\section{Computer Simulation of Hemispherical Punch Stretching}

For numerical experiments the LS-DYNA commercial code was used (see Fig. 4). The yield function (6) with different parameters $R$ was included as the original user's subroutine. In Fig. 3 the results of computer experiments are shown where curves 2 and 3 correspond to the parameters $R=2.0$ and $R=5.0$ respectively. From numerical experiments it follows that the parameter of anisotropy $R \approx 4.6$ for Hylite $\mathrm{H} 1$ and $R \approx 4.8$ for Hylite $\mathrm{H} 2$. For these parameters both curves coincide with curves 1 in both Fig. 4.a and 4.b. 


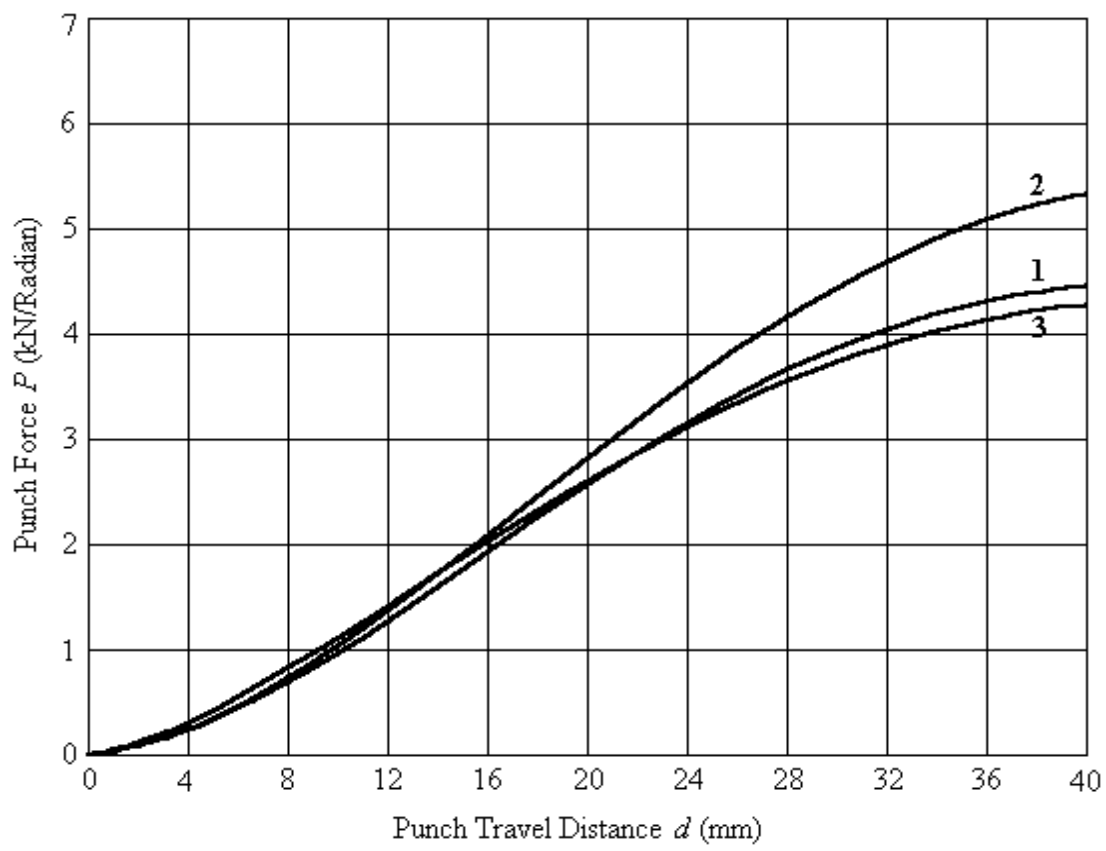

a.

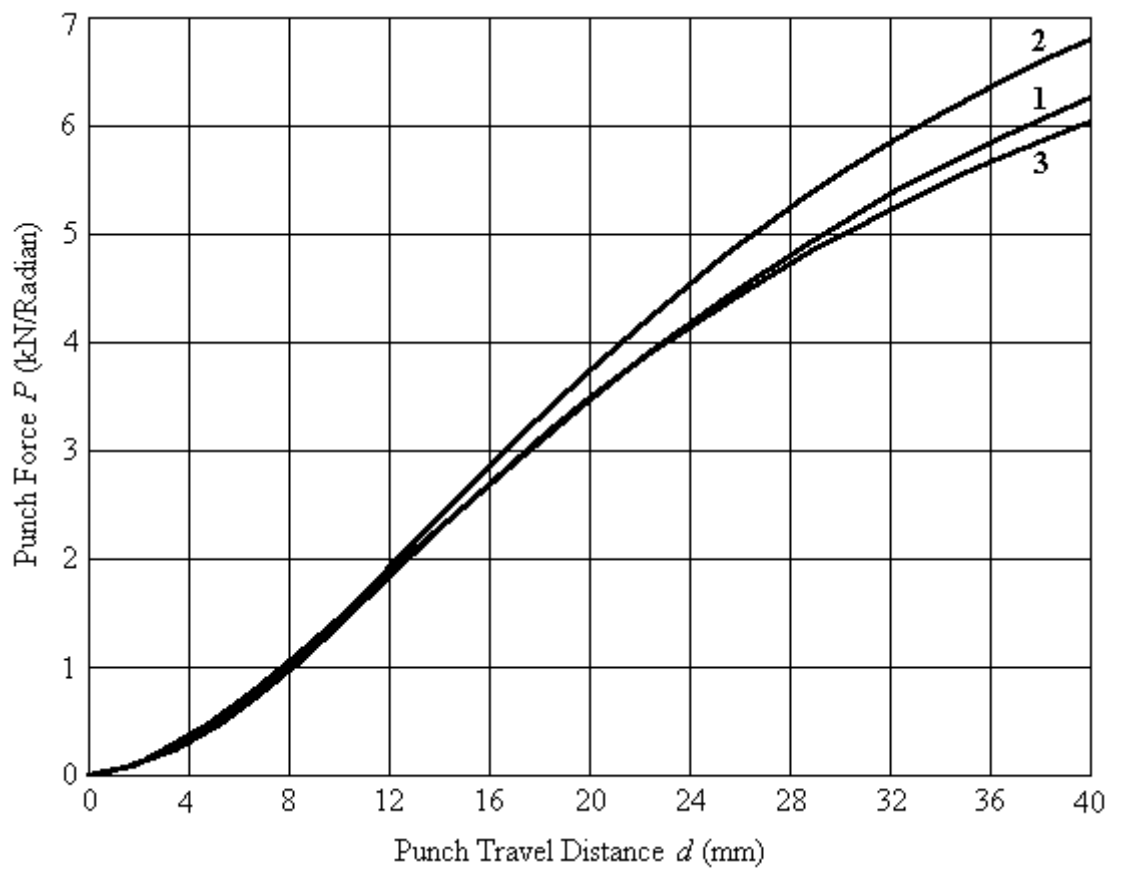

b.

Figure 3: Comparison of the punch force per unit radian:

(a) corresponds to Hylite H1, (b) corresponds to Hylite H2. 


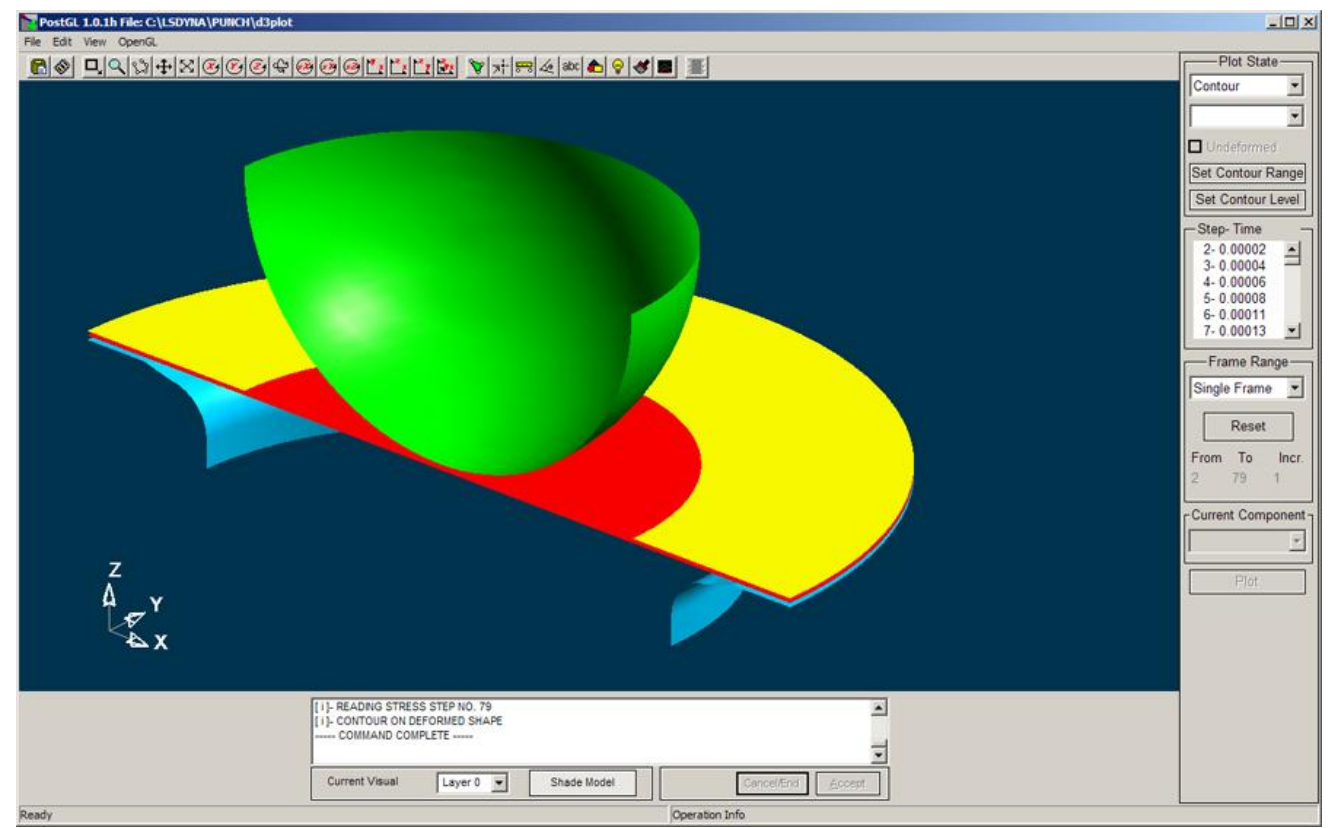

a.

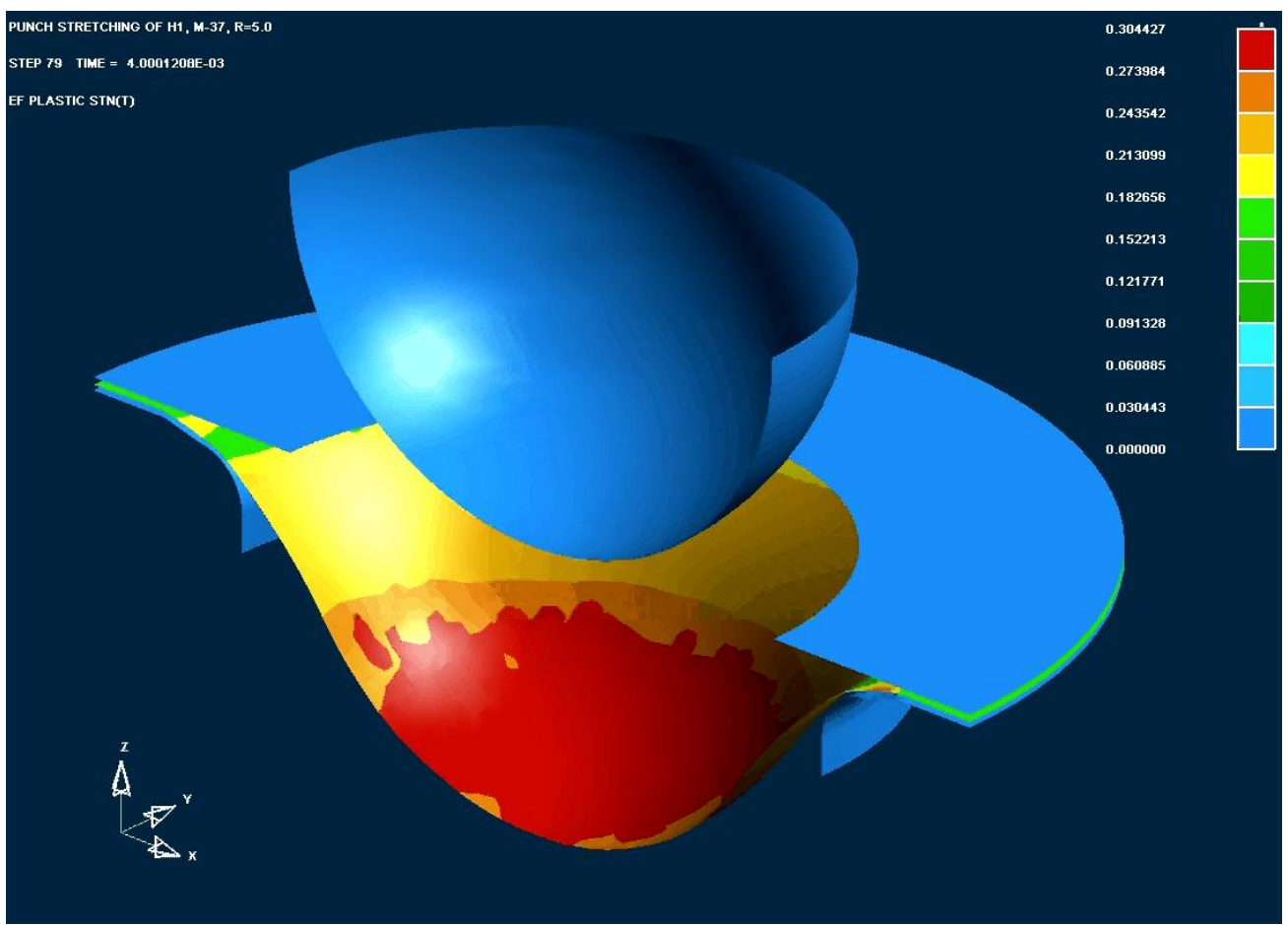

b.

Figure 4: The hemispherical punch stretching operation of Hylite by LS-DYNA cod: (a) describes the initial configuration,

(b) describes the final configuration after stamping. 


\section{Conclusion}

The results of the computer simulation are demonstrated that the constitutive relation (6) can be effectively used for modeling of forming processes for sandwich sheets Hylites used in car industry. For example, some parts of the car Ford Focus III are prepared from the sandwich material Hylite H3.

\section{Acknowledgements}

The work was supported by the Ford Motor Company within a framework of University Research Program and was partially supported by the German Academic Exchange Service (DAAD) in 2012 during a scientific work at the Otto-von-Guericke University Magdeburg.

\section{References}

[1] G.A. Holzapfel, Nonlinear Solid Mechanics: A Continuum Approach for Engineering, John Wiley, Chichester, 2000.

[2] A.A. Iljushin, Finite relations between forces and moments and their connection with deformations in the shell theory, Appl. Math. Mech. 19 (1) (1945) 28-43.

[3] R. Hill, Classical plasticity: a retrospective view and a new proposal, J. Mech. Phys. Solids 42(11) (1994) 1803-1816.

[4] V.V. Novozilov, Yu.I. Kadashevitch, Micro-stresses into design materials, Mashinostroyeniye, Leningrad, 1990 (in Russian).

[5] S.N Atluri, On constitutive relations at finite strains: Hypo-elasticity and elasto-plastisity with isotropic or kinematic hardening, Computational Methods in Appl. Mech. Eng. 43(2) (1984) 137-171.

[6] I.A. Brigadnov, Large elasto-plastic strains of composites, Voprosi Materialovedeniya 33(1) (2003) 411-417. 